\title{
ON THE CAUCHY PROBLEM FOR THE HARTREE TYPE EQUATION IN THE WIENER ALGEBRA
}

\author{
RÉMI CARLES AND LOUNÈS MOUZAOUI \\ (Communicated by James E. Colliander)
}

\begin{abstract}
We consider the mass-subcritical Hartree equation with a homogeneous kernel in the space of square integrable functions whose Fourier transform is integrable. We prove a global well-posedness result in this space. On the other hand, we show that the Cauchy problem is not even locally wellposed if we simply work in the space of functions whose Fourier transform is integrable. Similar results are proven when the kernel is not homogeneous and is such that its Fourier transform belongs to some Lebesgue space.
\end{abstract}

\section{INTRODUCTION}

We consider the Cauchy problem for the Hartree equation

$$
i \partial_{t} u+\Delta u=\left(K *|u|^{2}\right) u, \quad t \in \mathbf{R}, x \in \mathbf{R}^{d} ; \quad u_{\mid t=0}=u_{0},
$$

where $K$ denotes the Hartree kernel. We first deal with the case of a homogeneous kernel,

$$
K(x)=\frac{\lambda}{|x|^{\gamma}}, \quad \lambda \in \mathbf{R}, \gamma>0 .
$$

In [21], it was proved that if $1 \leqslant d \leqslant 3$ and $\gamma<d$, the Cauchy problem (1.1) is locally well-posed in $L^{2}\left(\mathbf{R}^{d}\right) \cap W$, where $W$ stands for the Wiener algebra (also called Fourier algebra, according to the context)

$$
W=\left\{f \in \mathcal{S}^{\prime}\left(\mathbf{R}^{d} ; \mathbf{C}\right), \quad\|f\|_{W}:=\|\widehat{f}\|_{L^{1}\left(\mathbf{R}^{d}\right)}<\infty\right\},
$$

and the Fourier transform is defined, for $f \in L^{1}\left(\mathbf{R}^{d}\right)$, as

$$
\widehat{f}(\xi)=\frac{1}{(2 \pi)^{d / 2}} \int_{\mathbf{R}^{d}} e^{-i x \cdot \xi} f(x) \mathrm{d} x .
$$

A motivation for working in $W$ lies in the fact that $W \hookrightarrow L^{\infty}\left(\mathbf{R}^{d}\right)$, and, contrary to e.g. $H^{s}\left(\mathbf{R}^{d}\right), s>d / 2, W$ scales like $L^{\infty}\left(\mathbf{R}^{d}\right)$ (note also that if $s>d / 2$, $\left.H^{s}\left(\mathbf{R}^{d}\right) \hookrightarrow W\right)$. So in a way, $W$ is the largest space included in $L^{\infty}\left(\mathbf{R}^{d}\right)$ on which the Schrödinger group $e^{i t \Delta}$ acts continuously. Recall that $e^{i t \Delta}$ does not map $L^{\infty}\left(\mathbf{R}^{d}\right)$ to itself, as shown by the explicit formula $e^{i \Delta}\left(e^{-i|x|^{2} / 4}\right)=\delta_{x=0}$ and the parabolic scale invariance.

Received by the editors May 16, 2012 and, in revised form, July 17, 2012 and August 1, 2012. 2010 Mathematics Subject Classification. Primary 35Q55; Secondary 35A01, 35B30, 35B45, $35 \mathrm{~B} 65$.

Key words and phrases. Hartree equation, well-posedness, Wiener algebra.

This work was supported by the French ANR project R.A.S. (ANR-08-JCJC-0124-01). 
The other motivation for this study is that Wiener algebra has recently proved useful in the description of high frequency regimes. After the first step was accomplished in [16, it was confirmed in [9, 19] that this analytical framework is very useful in the mathematical theory of geometric optics in the context of semilinear hyperbolic equations. In [4, similar results were obtained in the case of Schrödinger equations with a power-like nonlinearity (mostly cubic) of the form

$$
i \varepsilon \partial_{t} u^{\varepsilon}+\varepsilon^{2} \Delta u^{\varepsilon}=\varepsilon\left|u^{\varepsilon}\right|^{2} u^{\varepsilon} .
$$

The scaling in $\varepsilon$ makes it difficult (if not impossible) to use Strichartz estimates to justify asymptotic expansions as $\varepsilon \rightarrow 0$. So $L^{\infty}$-estimates are needed in the nonlinear analysis, and working in the Wiener algebra seems to be the cheapest way to obtain such estimates. We have the general estimate

$$
\left\|g^{\varepsilon}\right\|_{L^{\infty}} \leqslant(2 \pi)^{-d / 2}\left\|g^{\varepsilon}\right\|_{W}
$$

hence an $L^{\infty}$-bound which is independent of $\varepsilon$, provided that uniform estimates are available in $W$. On the other hand, if one works in Sobolev spaces, then the following estimate is sharp in terms of powers of $\varepsilon$ (see e.g. [23]): for $s>d / 2$,

$$
\left\|g^{\varepsilon}\right\|_{L^{\infty}} \leqslant C \varepsilon^{-d / 2}\left(\left\|g^{\varepsilon}\right\|_{L^{2}}+\left\|(\varepsilon D)^{s} g^{\varepsilon}\right\|_{L^{2}}\right)
$$

where $C$ is independent of $\varepsilon$ and $D=(-\Delta)^{1 / 2}$. For functions of the form $a(x) e^{i k \cdot x / \varepsilon}$, $k \in \mathbf{R}^{d} \backslash\{0\}$, the right hand side will be of order exactly $\varepsilon^{-d / 2}$. As a consequence, to justify asymptotic expansions in Sobolev spaces in such a context, it is necessary to construct asymptotic expansions to a rather high order to compensate for the singular factor $\varepsilon^{-d / 2}$ in the above Sobolev embedding (the error has to be at least $\mathcal{O}\left(\varepsilon^{d / 2}\right)$ in the rescaled Sobolev space $\left.H_{\varepsilon}^{s}, s>d / 2\right)$. The case of Schrödinger equations with a nonlocal nonlinearity as in (1.1) was considered first in [13] for kernels $K$ such that $(1+|\xi|) \widehat{K}(\xi) \in L^{\infty}\left(\mathbf{R}^{d}\right)$, and then in [5] for Davey-Stewartson nonlinearity, where one only has $\widehat{K} \in L^{\infty}\left(\mathbf{R}^{d}\right)$, but $\widehat{K}$ is 0 -homogeneous. In all these references, the Wiener regularity was enough to construct local and approximate solutions, and to establish error estimates. On the other hand, in the case where $K$ is of the form (1.2), the high frequency analysis in 21] was performed in $L^{2} \cap W$, and not only in $W$. The results of the present paper show that working in $L^{2} \cap W$ instead of $W$ is not only convenient in order to justify an asymptotic expansion, it is also necessary to have a reasonable theory for the Cauchy problem.

First, we prove that if $d \geqslant 1$ and $\gamma<\min (2, d / 2)$, then the solution to (1.1) is global in time in $L^{2} \cap W$. In view of the classical result according to which (1.1) is globally well-posed in $L^{2}\left(\mathbf{R}^{d}\right)$, our result can be understood as a propagation of the Wiener regularity. On the other hand, the mere Wiener regularity does not suffice to ensure even local well-posedness for (1.1).

Theorem 1.1. Let $d \geqslant 1, K$ be given by (1.2) with $\lambda \in \mathbf{R}$ and $0<\gamma<\min (2, d / 2)$. If $u_{0} \in L^{2}\left(\mathbf{R}^{d}\right) \cap W$, then (1.1) has a unique, global solution $u \in C\left(\mathbf{R} ; L^{2} \cap W\right)$. In addition, its $L^{2}$-norm is conserved,

$$
\|u(t)\|_{L^{2}}=\left\|u_{0}\right\|_{L^{2}}, \quad \forall t \in \mathbf{R} .
$$

Remark 1.2. One might be tempted to consider a space included in $L^{\infty}$ which also scales like $L^{\infty}$ and which is larger than $W=\mathcal{F} L^{1}$, namely the amalgam space $W\left(\mathcal{F} L^{1} ; L^{\infty}\right)$. This space consists essentially of functions which are locally in $W$, and globally in $L^{\infty}$ (see e.g. [11, 12 for a precise definition). Strichartz estimates in amalgam spaces have been established in [11] (even though the case 
$W\left(\mathcal{F} L^{1} ; L^{\infty}\right)$ can never be considered). However, since the map $x \mapsto e^{i|x|^{2}}$ belongs to $W\left(\mathcal{F} L^{1} ; L^{\infty}\right)$, we see that $e^{i t \Delta}$ does not act continuously on $W\left(\mathcal{F} L^{1} ; L^{\infty}\right)$.

In 21, it was convenient to work in the space $L^{2} \cap W$ instead of merely $W$, in order to describe the high frequency regime for nonlocal nonlinear Schrödinger type equations. The following result shows that it was not only convenient, but necessary: (1.1) is not well-posed in the mere Wiener algebra.

Theorem 1.3. Let $d \geqslant 1$, and let $K$ be given by (1.2) with $0<\gamma<d$. The Cauchy problem (1.1) is locally well-posed in $W \cap L^{2}$, but not in $W$ : for all balls $B$ of $W$, for all $T>0$ the solution map $\varphi \in B \mapsto \psi \in C([0, T] ; W)$ is not uniformly continuous.

Remark 1.4. In the case of the nonlinear Schrödinger equation

$$
i \partial_{t} u+\Delta u=\lambda|u|^{2 \sigma} u,
$$

where $\sigma$ is an integer and $\lambda \in \mathbf{R}$, the Cauchy problem is locally well-posed in $W$ (see [4]). From the above result, this is in sharp contrast with the case of the Hartree equation. On the other hand, it is not clear that the Cauchy problem for (1.3) is globally well-posed in $L^{2}\left(\mathbf{R}^{d}\right) \cap W$, even in the case $d=\sigma=1$. We note that in [15], the authors study (1.3) in the one-dimensional case $d=1$, with $\sigma<2$ not necessarily an integer. They prove local well-posedness in

$$
\hat{L}^{p}=\left\{f: \hat{f} \in L^{p^{\prime}}\right\}
$$

for $p$ in some open neighborhood of 2 . Global well-posedness results for initial data in $\hat{L}^{p}$ are established in spaces based on dispersive estimates.

The kernel $K$ given by (1.2) is such that its Fourier transform does not belong to a Lebesgue space, but to a weak Lebesgue space, from the following property (see e.g. [1, Proposition 1.29]):

Proposition 1.5. Let $d \geqslant 1$ and $0<\gamma<d$. There exists $C=C(\gamma, d)$ such that the Fourier transform of $K$ defined by (1.2) is

$$
\widehat{K}(\xi)=\frac{\lambda C}{|\xi|^{d-\gamma}} .
$$

The final result of this paper is concerned with the case where the kernel $K$ is such that its Fourier transform belongs to some Lebesgue space. It is a natural generalization of Theorem 1.3 and is also related to the framework of Davey-Stewartson equations, as studied in [5]. The general assumption on $K$ in $\left[5\right.$ ] is that $\widehat{K} \in L^{\infty}$, with $\widehat{K}$ 0-homogeneous, and continuous outside the origin: if $\widehat{K} \in L^{p} \backslash L^{\infty}$ for some finite $p$, then the local Cauchy problem may not be well-posed in $W$.

Theorem 1.6. Let $d \geqslant 1$.

- Let $p \in[1, \infty]$, and suppose that $K$ is such that $\widehat{K} \in L^{p}\left(\mathbf{R}^{d}\right)$. If $u_{0} \in$ $L^{2}\left(\mathbf{R}^{d}\right) \cap W$, then there exists $T>0$ such that (1.1) has a unique solution $u \in C\left([-T, T] ; L^{2} \cap W\right)$. In addition, its $L^{2}$-norm is conserved,

$$
\|u(t)\|_{L^{2}}=\left\|u_{0}\right\|_{L^{2}}, \quad \forall t \in[-T, T] .
$$

If $K \in W(p=1)$, then the solution is global: $u \in C\left(\mathbf{R} ; L^{2} \cap W\right)$.

- Suppose that $K$ is such that $\widehat{K} \in L^{\infty}\left(\mathbf{R}^{d}\right)$. If $u_{0} \in W$, then there exists $T>0$ such that (1.1) has a unique solution $u \in C([-T, T] ; W)$. 
- For any $p \in[1, \infty)$, one can find $K$ with $\widehat{K} \in L^{p}\left(\mathbf{R}^{d}\right) \backslash L^{\infty}\left(\mathbf{R}^{d}\right)$, such that (1.1) is locally well-posed in $L^{2}\left(\mathbf{R}^{d}\right) \cap W$, but not in $W$ : for all balls $B$ of $W$, for all $T>0$ the solution map $\varphi \in B \mapsto \psi \in C([0, T] ; W)$ is not uniformly continuous.

Remark 1.7. Other nonlocal nonlinearities have been considered. We mention one which is related to the present study, since it is motivated by local well-posedness issues. In [7, 10, 22], the authors have considered the so-called Wick ordered cubic NLS,

$$
i \partial_{t} u+\partial_{x}^{2} u= \pm\left(|u|^{2}-2 \int|u|^{2}\right) u,
$$

for $x \in \mathbf{T}$. The effect of removing the zero Fourier mode is to partially neutralize the instability phenomenon due to the transfer from high to low frequencies described in $3,4,8$, a phenomenon which is rather similar to the one used in the present paper to prove Theorem 1.3 and the last point in Theorem 1.6. Indeed, weaker regularity is allowed for (1.4). Typically, the weak $L^{2}$ continuity holds for (1.4), while it does not for NLS [20].

\section{Standard Existence Results and properties}

2.1. Main properties of the Wiener algebra. The space $W$ enjoys the following elementary properties (see [4, 9]):

(1) $W$ is a Banach space, continuously embedded into $L^{\infty}\left(\mathbf{R}^{d}\right)$.

(2) $W$ is an algebra, and the mapping $(f, g) \mapsto f g$ is continuous from $W^{2}$ to $W$, with

$$
\|f g\|_{W} \leqslant\|f\|_{W}\|g\|_{W}, \quad \forall f, g \in W .
$$

(3) For all $t \in \mathbf{R}$, the free Schrödinger group $e^{i t \Delta}$ is unitary on $W$.

2.2. Existence results based on Strichartz estimates. For the sake of completeness, we recall the standard definition and results.

Definition 2.1. A pair $(p, q) \neq(2, \infty)$ is admissible if $p \geqslant 2, q \geqslant 2$, and

$$
\frac{2}{p}=d\left(\frac{1}{2}-\frac{1}{q}\right) \text {. }
$$

Proposition 2.2 (From [14,18]). (1) For any admissible pair $(p, q)$, there exists $C_{q}$ such that

$$
\left\|e^{i t \Delta} \varphi\right\|_{L^{p}\left(\mathbf{R} ; L^{q}\right)} \leqslant C_{q}\|\varphi\|_{L^{2}}, \quad \forall \varphi \in L^{2}\left(\mathbf{R}^{d}\right)
$$

(2) Denote

$$
D(F)(t, x)=\int_{0}^{t} e^{i(t-\tau) \Delta} F(\tau, x) \mathrm{d} \tau .
$$

For all admissible pairs $\left(p_{1}, q_{1}\right)$ and $\left(p_{2}, q_{2}\right)$, there exists $C=C_{q_{1}, q_{2}}$ such that for all intervals $I \ni 0$,

$$
\|D(F)\|_{L^{p_{1}\left(I ; L^{q_{1}}\right)}} \leqslant C\|F\|_{L^{p_{2}^{\prime}}\left(I ; L^{q_{2}^{\prime}}\right)}, \quad \forall F \in L^{p_{2}^{\prime}}\left(I ; L^{q_{2}^{\prime}}\right) .
$$


Proposition 2.3. Let $d \geqslant 1, K$ be given by (1.2) with $\lambda \in \mathbf{R}$ and $0<\gamma<$ $\min (2, d)$. If $u_{0} \in L^{2}\left(\mathbf{R}^{d}\right)$, then (1.1) has a unique, global solution

$$
u \in C\left(\mathbf{R} ; L^{2}\right) \cap L_{\mathrm{loc}}^{8 / \gamma}\left(\mathbf{R} ; L^{4 d /(2 d-\gamma)}\right) .
$$

In addition, its $L^{2}$-norm is conserved,

$$
\|u(t)\|_{L^{2}}=\left\|u_{0}\right\|_{L^{2}}, \quad \forall t \in \mathbf{R},
$$

and for all admissible pairs $(p, q), u \in L_{\mathrm{loc}}^{p}\left(\mathbf{R} ; L^{q}\left(\mathbf{R}^{d}\right)\right)$.

Proof. We give the main technical steps of the proof and refer to [6] for details. By Duhamel's formula, we write (1.1) as

$$
u(t)=e^{i t \Delta} u_{0}-i \int_{0}^{t} e^{i(t-\tau) \Delta}\left(K *|u|^{2} u\right)(\tau) \mathrm{d} \tau=: \Phi(u)(t) .
$$

Introduce the space

$$
\begin{gathered}
Y(T)=\left\{\phi \in C\left([0, T] ; L^{2}\left(\mathbf{R}^{d}\right)\right):\|\phi\|_{L^{\infty}\left([0, T] ; L^{2}\left(\mathbf{R}^{d}\right)\right)} \leqslant 2\left\|u_{0}\right\|_{L^{2}\left(\mathbf{R}^{d}\right)},\right. \\
\left.\|\phi\|_{L^{8 / \gamma}\left([0, T] ; L^{4 d /(2 d-\gamma)}\left(\mathbf{R}^{d}\right)\right)} \leqslant 2 C(8 / \gamma)\left\|u_{0}\right\|_{L^{2}\left(\mathbf{R}^{d}\right)}\right\},
\end{gathered}
$$

and the distance

$$
d\left(\phi_{1}, \phi_{2}\right)=\left\|\phi_{1}-\phi_{2}\right\|_{L^{8 / \gamma}\left([0, T] ; L^{4 d /(2 d-\gamma)}\right)},
$$

where $C(8 / \gamma)$ stems from Proposition 2.2. Then $(Y(T), d)$ is a complete metric space, as remarked in [17] (see also [6]). Hereafter, we denote by

$$
q=\frac{8}{\gamma}, \quad r=\frac{4 d}{2 d-\gamma}, \quad \theta=\frac{8}{4-\gamma},
$$

and $\|\cdot\|_{L^{a}\left([0, T] ; L^{b}\left(\mathbf{R}^{d}\right)\right)}$ by $\|\cdot\|_{L^{a} L^{b}}$ for simplicity. Notice that $(q, r)$ is admissible and

$$
\frac{1}{q^{\prime}}=\frac{4-\gamma}{4}+\frac{1}{q}=\frac{1}{2}+\frac{1}{\theta} \quad ; \quad \frac{1}{r^{\prime}}=\frac{\gamma}{2 d}+\frac{1}{r} \quad ; \quad \frac{1}{2}=\frac{1}{\theta}+\frac{1}{q} .
$$

By using Strichartz estimates, Hölder inequality and Hardy-Littlewood-Sobolev inequality, we have, for $(\underline{q}, \underline{r}) \in\{(q, r),(\infty, 2)\}$ :

$$
\begin{aligned}
\|\Phi(u)\|_{L^{\underline{q}} L_{\underline{r}}} & \leqslant C(\underline{q})\left\|u_{0}\right\|_{L^{2}}+C(\underline{q}, q)\left\|\left(K *|u|^{2}\right) u\right\|_{L^{q^{\prime} L^{r^{\prime}}}} \\
& \leqslant C(\underline{q})\left\|u_{0}\right\|_{L^{2}}+C(\underline{q}, q)\left\|K *|u|^{2}\right\|_{L^{4 /(4-\gamma)} L^{2 d / \gamma}}\|u\|_{L^{q} L^{r}} \\
& \leqslant C(\underline{q})\left\|u_{0}\right\|_{L^{2}}+C\|u\|_{L^{\theta} L^{r}}^{2}\|u\|_{L^{q} L^{r}} \\
& \leqslant C(\underline{q})\left\|u_{0}\right\|_{L^{2}}+C T^{1-\gamma / 2}\|u\|_{L^{q} L^{r}}^{3},
\end{aligned}
$$

for any $u \in Y(T)$, with $C(\infty)=1$ by the standard energy estimate. To show the contraction property of $\Phi$, for any $v, w \in Y(T)$, we get

$$
\begin{aligned}
\|\Phi(v)-\Phi(w)\|_{L^{q} L^{r}} \lesssim & \left\|K *|v|^{2}\right\|_{L^{4 /(4-\gamma)} L^{2 d / \gamma}}\|v-w\|_{L^{q} L^{r}} \\
& +\left\|\left.K *|| v\right|^{2}-|w|^{2}\right\|\left\|_{L^{2} L^{2 d / \gamma}}\right\| w \|_{L^{\theta} L^{r}} \\
\lesssim & \left.\|v\|_{L^{\theta} L^{r}}^{2}+\|w\|_{L^{\theta} L^{r}}^{2}\right)\|v-w\|_{L^{q} L^{r}} \\
\leqslant & C T^{1-\gamma / 2}\left(\|v\|_{L^{q} L^{r}}^{2}+\|w\|_{L^{q} L^{r}}^{2}\right)\|v-w\|_{L^{q} L^{r}} .
\end{aligned}
$$

Thus $\Phi$ is a contraction from $Y(T)$ to $Y(T)$ provided that $T$ is sufficiently small. Then there exists a unique $u \in Y(T)$ solving (1.1). The global existence of the solution for (1.1) follows from the conservation of the $L^{2}$-norm of $u$. The last 
property of the proposition then follows from Strichartz estimates applied with an arbitrary admissible pair on the left hand side and the same pairs as above on the right hand side.

\section{Proof of Theorem 1.1}

Throughout this section, we assume that the kernel $K$ is given by (1.2).

3.1. Uniqueness. Uniqueness stems from the local well-posedness result established in [21, based on the following lemma, whose proof is recalled for the sake of completeness.

Lemma 3.1. Let $0<\gamma<d$. There exists $C$ such that for all $f, g \in L^{2}\left(\mathbf{R}^{d}\right) \cap W$,

$$
\left\|\left(K *|f|^{2}\right) f-\left(K *|g|^{2}\right) g\right\|_{L^{2} \cap W} \leqslant C\left(\|f\|_{L^{2} \cap W}^{2}+\|g\|_{L^{2} \cap W}^{2}\right)\|f-g\|_{L^{2} \cap W} .
$$

Proof. Let $\kappa_{1}=\mathbf{1}_{\{|\xi| \leqslant 1\}} \widehat{K}$ and $\kappa_{2}=\mathbf{1}_{\{|\xi|>1\}} \widehat{K}$. In view of Proposition 1.5, $\kappa_{1} \in$ $L^{p}\left(\mathbf{R}^{d}\right)$ for all $p \in\left[1, \frac{d}{d-\gamma}\right)$ and $\kappa_{2} \in L^{q}\left(\mathbf{R}^{d}\right)$ for all $q \in\left(\frac{d}{d-\gamma}, \infty\right]$. For $h \in$ $L^{1}\left(\mathbf{R}^{d}\right) \cap W$, we have

$$
\begin{aligned}
\|K * h\|_{W} & \lesssim\left\|\kappa_{1} \widehat{h}\right\|_{L^{1}}+\left\|\kappa_{2} \widehat{h}\right\|_{L^{1}} \lesssim\left\|\kappa_{1}\right\|_{L^{1}}\|\widehat{h}\|_{L^{\infty}}+\left\|\kappa_{2}\right\|_{L^{\infty}}\|\widehat{h}\|_{L^{1}} \\
& \lesssim\|h\|_{L^{1}}+\|\widehat{h}\|_{L^{1}},
\end{aligned}
$$

where we have used Hausdorff-Young inequality. Writing

$$
\left(K *|f|^{2}\right) f-\left(K *|g|^{2}\right) g=\left(K *|f|^{2}\right)(f-g)+\left(K *\left(|f|^{2}-|g|^{2}\right)\right) g,
$$

the lemma follows, since $W$ is a Banach algebra embedded into $L^{\infty}\left(\mathbf{R}^{d}\right)$.

We infer uniqueness in $L^{2}\left(\mathbf{R}^{d}\right) \cap W$ for (1.1) as soon as $0<\gamma<d$ :

Proposition 3.2. Let $0<\gamma<d, T>0$, and $u, v \in C\left([0, T] ; L^{2} \cap W\right)$ solve (1.1), with the same initial datum $u_{0} \in L^{2}\left(\mathbf{R}^{d}\right) \cap W$. Then $u \equiv v$.

Proof. Duhamel's formula yields

$$
u(t)-v(t)=-i \int_{0}^{t} e^{i(t-\tau) \Delta}\left(\left(K *|u|^{2}\right) u-\left(K *|v|^{2}\right) v\right)(\tau) \mathrm{d} \tau .
$$

Since the Schrödinger group is unitary on $L^{2}$ and on $W$, Minkowski inequality and Lemma 3.1 yield, for $t \geqslant 0$,

$$
\begin{aligned}
\|u(t)-v(t)\|_{L^{2} \cap W} \lesssim \int_{0}^{t}\left(\|u(\tau)\|_{L^{2} \cap W}^{2}+\|v(\tau)\|_{L^{2} \cap W}^{2}\right)\|u(\tau)-v(\tau)\|_{L^{2} \cap W} \mathrm{~d} \tau \\
\lesssim\left(\|u\|_{L^{\infty}\left([0, T] ; L^{2} \cap W\right)}^{2}+\|v\|_{L^{\infty}\left([0, T] ; L^{2} \cap W\right)}^{2}\right) \int_{0}^{t}\|u(\tau)-v(\tau)\|_{L^{2} \cap W} \mathrm{~d} \tau
\end{aligned}
$$

The Gronwall lemma implies $u \equiv v$.

3.2. Existence. In view of Lemma 3.1, the standard fixed point argument yields:

Proposition 3.3. Let $d \geqslant 1, \lambda \in \mathbf{R}, 0<\gamma<d$, and $K$ be given by (1.2). If $u_{0} \in L^{2}\left(\mathbf{R}^{d}\right) \cap W$, then there exists $T>0$ depending only on $\lambda, \gamma, d$ and $\left\|u_{0}\right\|_{L^{2} \cap W}$, and a unique $u \in C\left([0, T] ; L^{2} \cap W\right)$ to (1.1). 
Taking Proposition 2.3 into account, to establish Theorem 1.1, it suffices to prove that the Wiener norm of $u$ cannot become unbounded in finite time.

Resuming the decomposition of $\widehat{K}$ introduced in the proof of Lemma 3.1, we find

$$
\begin{aligned}
\|u(t)\|_{W} & \leqslant\left\|u_{0}\right\|_{W}+\int_{0}^{t}\left\|\left(K *|u(\tau)|^{2}\right) u(\tau)\right\|_{W} \mathrm{~d} \tau \\
& \leqslant\left\|u_{0}\right\|_{W}+\int_{0}^{t}\left\|K *|u(\tau)|^{2}\right\|_{W}\|u(\tau)\|_{W} \mathrm{~d} \tau \\
& \leqslant\left\|u_{0}\right\|_{W}+\int_{0}^{t}\left(\left\|\kappa_{1}\right\|_{L^{1}}\|u(\tau)\|_{L^{2}}^{2}+\left\|\kappa_{2}\right\|_{L^{p}}\left\|\widehat{|u(\tau)|^{2}}\right\|_{L^{p^{\prime}}}\right)\|u(\tau)\|_{W} \mathrm{~d} \tau
\end{aligned}
$$

provided that $p>\frac{d}{d-\gamma}$. Using the conservation of the $L^{2}$-norm of $u$ and HausdorffYoung inequality, we infer, if $p \leqslant 2$,

$$
\|u(t)\|_{W} \lesssim\left\|u_{0}\right\|_{W}+\int_{0}^{t}\left(\left\|\kappa_{1}\right\|_{L^{1}}\left\|u_{0}\right\|_{L^{2}}^{2}+\left\|\kappa_{2}\right\|_{L^{p}}\left\||u(\tau)|^{2}\right\|_{L^{p}}\right)\|u(\tau)\|_{W} \mathrm{~d} \tau .
$$

To summarize, for all $1<\frac{d}{d-\gamma}<p \leqslant 2$, there exists $C$ such that

$$
\|u(t)\|_{W} \leqslant\left\|u_{0}\right\|_{W}+C \int_{0}^{t}\|u(\tau)\|_{W} \mathrm{~d} \tau+C \int_{0}^{t}\|u(\tau)\|_{L^{2 p}}^{2}\|u(\tau)\|_{W} \mathrm{~d} \tau .
$$

The above requirement on $p$ can be fulfilled if and only if $0<\gamma<d / 2$. To take advantage of Proposition 2.3, introduce $\alpha>1$ such that $(2 \alpha, 2 p)$ is admissible. This is possible provided that $2 p<\frac{2 d}{d-2}$ when $d \geqslant 3$ : this condition is compatible with the requirement $p>\frac{d}{d-\gamma}$ if and only if $\gamma<2$. Using Hölder inequality for the last integral, we have

$$
\|u(t)\|_{W} \leqslant\left\|u_{0}\right\|_{W}+C \int_{0}^{t}\|u(\tau)\|_{W} \mathrm{~d} \tau+C\|u\|_{L^{2 \alpha}\left([0, t] ; L^{2 p}\right)}^{2}\|u\|_{L^{\alpha^{\prime}}([0, t] ; W)} .
$$

Set

$$
\omega(t)=\sup _{0 \leqslant \tau \leqslant t}\|u(\tau)\|_{W}
$$

For a given $T>0, \omega$ satisfies an estimate of the form

$$
\omega(t) \leqslant\left\|u_{0}\right\|_{W}+C \int_{0}^{t} \omega(\tau) \mathrm{d} \tau+C_{0}(T)\left(\int_{0}^{t} \omega(\tau)^{\alpha^{\prime}} \mathrm{d} \tau\right)^{1 / \alpha^{\prime}},
$$

provided that $0 \leqslant t \leqslant T$, and where we have used the fact that $\alpha^{\prime}$ is finite. Using Hölder inequality, we infer

$$
\omega(t) \leqslant\left\|u_{0}\right\|_{W}+C_{1}(T)\left(\int_{0}^{t} \omega(\tau)^{\alpha^{\prime}} \mathrm{d} \tau\right)^{1 / \alpha^{\prime}} .
$$

Raising the above estimate to the power $\alpha^{\prime}$, we find

$$
\omega(t)^{\alpha^{\prime}} \lesssim 1+\int_{0}^{t} \omega(\tau)^{\alpha^{\prime}} \mathrm{d} \tau
$$

The Gronwall lemma shows that $\omega \in L^{\infty}([0, T])$. Since $T>0$ is arbitrary, $\omega \in$ $L_{\mathrm{loc}}^{\infty}(\mathbf{R})$, and the result follows. 


\section{Ill-Posedness in the mere Wiener Algebra}

In this section we still assume that $K$ is given by (1.2). We show that the Cauchy problem (1.1) is ill-posed in the mere Wiener algebra, i.e. without including $L^{2}$. We recall the definition of well-posedness for the problem (1.1).

Definition 4.1. Let $\left(S,\|\cdot\|_{S}\right)$ be a Banach space of initial data and $\left(D,\|\cdot\|_{D}\right)$ be a Banach space of space-time functions. The Cauchy problem (1.1) is well-posed from $D$ to $S$ if, for all bounded subsets $B \subset D$, there exist $T>0$ and a Banach space $X_{T} \hookrightarrow C([0, T] ; S)$ such that:

(1) For all $\varphi \in B$, (1.1) has a unique solution $\psi \in X_{T}$ with $\left.\psi\right|_{t=0}=\varphi$.

(2) The mapping $B \ni \varphi \mapsto \psi \in C([0, T] ; S)$ is uniformly continuous.

Proof of Theorem 1.3. In view of [2, it suffices to prove that one term in the Picard iterations of $\Phi$ defined in Section 2 does not verify Definition 4.1. We argue by contradiction and assume that (1.1) is well-posed from $W$ to $W$. Then, let $T>0$ be the local time existence of the solution. We recall that for $0<\gamma<d$, (1.1) is wellposed from $W \cap L^{2}$ to $W \cap L^{2}$ (see [21, Theorem 2.1], recalled in Proposition 3.3). We define the following operator associated to the second Picard iterate:

$$
D(f)(t, x)=-i \int_{0}^{t} e^{i(t-\tau) \Delta}\left(K *|\psi|^{2} \psi\right)(\tau, x) \mathrm{d} \tau,
$$

where $\psi$ is the solution of the free equation

$$
i \partial_{t} \psi+\Delta \psi=0,\left.\quad \psi\right|_{t=0}=f,
$$

that is, $\psi(t)=e^{i t \Delta} f$. We denote $e^{i t \Delta} f=U(t) f$. By [2, Proposition 1] the operator $D$ is continuous from $W$ to $W$; that is,

$$
\|D(f)(t)\|_{W} \leqslant C\|f\|_{W}^{3}, \quad \forall t \in[0, T],
$$

for some positive constant $C$. Let $f \in \mathcal{S}\left(\mathbf{R}^{d}\right)$ be an element of the Schwartz space. We define a family of functions indexed by $h>0$ by

$$
f^{h}(x)=f(h x) .
$$

For all $h>0,\left\|f^{h}\right\|_{L^{2}}=\frac{1}{h^{d / 2}}\|f\|_{L^{2}}$, so for $h>0$ close to 0 the family $\left(f^{h}\right)_{h>0}$ leaves any compact of $L^{2}$. Remark that $\widehat{f^{h}}(\xi)=h^{-d} \widehat{f}\left(\frac{\xi}{h}\right)$ and $\left\|f^{h}\right\|_{W}=\|f\|_{W}<\infty$, so (4.1) yields

$$
\left\|D\left(f^{h}\right)(t)\right\|_{W} \leqslant C\|f\|_{W}^{3} .
$$

We develop the expression of $\left\|D\left(f^{h}\right)(t)\right\|_{W}$. We have

$$
\begin{aligned}
& \mathcal{F}\left(K *\left|U(\tau) f^{h}\right|^{2} U(\tau) f^{h}\right)(\xi) \\
& =(2 \pi)^{d / 2} \mathcal{F}\left(K *\left|U(\tau) f^{h}\right|^{2}\right) * \mathcal{F}\left(U(\tau) f^{h}\right)(\xi) \\
& =(2 \pi)^{d / 2} \int_{\mathbf{R}^{d}} \mathcal{F}\left(K *\left|U(\tau) f^{h}\right|^{2}\right)(\xi-y) \mathcal{F}\left(U(\tau) f^{h}\right)(y) \mathrm{d} y \\
& =\int_{\mathbf{R}^{d}} e^{i \tau|y|^{2}} \widehat{K}(\xi-y) \mathcal{F}\left(\left|U(\tau) f^{h}\right|^{2}\right)(\xi-y) \widehat{f^{h}}(y) \mathrm{d} y \\
& =(2 \pi)^{d / 2} \iint_{\mathbf{R}^{2 d}} e^{i \tau|y|^{2}} e^{i \tau|\xi-y-z|^{2}} e^{-i \tau|z|^{2}} \widehat{K}(\xi-y) \widehat{f^{h}}(\xi-y-z) \widehat{f^{h}}(z) \widehat{f^{h}}(y) \mathrm{d} y \mathrm{~d} z \\
& =\frac{(2 \pi)^{d / 2}}{h^{3 d}} \iint_{\mathbf{R}^{2 d}} e^{i \tau|y|^{2}} e^{i \tau|\xi-y-z|^{2}} e^{-i \tau|z|^{2}} \widehat{K}(\xi-y) \widehat{f}\left(\frac{\xi-y-z}{h}\right) \widehat{\bar{f}}\left(\frac{z}{h}\right) \widehat{f}\left(\frac{y}{h}\right) \mathrm{d} y \mathrm{~d} z .
\end{aligned}
$$


Taking the $W$-norm gives

$$
\begin{aligned}
\left\|D\left(f^{h}\right)(t)\right\|_{W} & =\left\|\widehat{D\left(f^{h}\right)}(t)\right\|_{L^{1}} \\
& =\int_{\mathbf{R}^{d}}\left|\int_{0}^{t} \mathcal{F}\left(U(t-\tau)\left(K *\left|U(\tau) f^{h}\right|^{2} U(\tau) f^{h}\right)\right)(\xi) \mathrm{d} \tau\right| \mathrm{d} \xi \\
& =\int_{\mathbf{R}^{d}}\left|\int_{0}^{t} e^{i(t-\tau)|\xi|^{2}} \mathcal{F}\left(K *\left|U(\tau) f^{h}\right|^{2} U(\tau) f^{h}\right)(\xi) \mathrm{d} \tau\right| \mathrm{d} \xi .
\end{aligned}
$$

We replace $\mathcal{F}\left(K *\left|U(\tau) f^{h}\right|^{2} U(\tau) f^{h}\right)(\xi)$ by its integral formula above and apply the following changes of variable: $\xi^{\prime}=\xi / h, y^{\prime}=y / h, z^{\prime}=z / h, \tau^{\prime}=\tau h^{2}$. We obtain

$$
\left\|D\left(f^{h}\right)(t)\right\|_{W}=\frac{1}{h^{d-\gamma+2}}\left\|D(f)\left(t h^{2}\right)\right\|_{W} .
$$

Let $s \in(0, T)$. We examine more closely the term $F(s):=\int_{0}^{s} U(s-\tau) g(\tau) \mathrm{d} \tau$ where $g(s):=\left(K *|U(s) f|^{2}\right) U(s) f$. The Taylor formula yields

$$
F(s)=F(0)+F^{\prime}(0) s+\frac{s^{2}}{2} \int_{0}^{1}(1-\theta) F^{\prime \prime}(s \theta) \mathrm{d} \theta .
$$

We have $F(0)=0$, so for $s \in[0,1]$,

$$
\begin{aligned}
\left\|F(s)-F^{\prime}(0) s\right\|_{W} & \leqslant s^{2}\left\|\int_{0}^{1}(1-\theta) F^{\prime \prime}(s \theta) \mathrm{d} \theta\right\|_{W} \mathrm{~d} \theta \\
& \leqslant s^{2} \int_{0}^{1}\left\|F^{\prime \prime}(s \theta)\right\|_{W} \mathrm{~d} \theta \leqslant s^{2}\left\|F^{\prime \prime}\right\|_{L^{\infty}([0,1] ; W)} .
\end{aligned}
$$

The first and second derivatives of $F$ are given by

$$
\begin{aligned}
F^{\prime}(s) & =g(s)+\int_{0}^{s} U(s-\tau) i \Delta g(\tau) \mathrm{d} \tau \\
F^{\prime \prime}(s) & =g^{\prime}(s)+i \Delta g(s)-\int_{0}^{s} U(s-\tau) \Delta^{2} g(\tau) \mathrm{d} \tau,
\end{aligned}
$$

so $F^{\prime}(0)=g(0)$ and

$$
\left\|F^{\prime \prime}\right\|_{L^{\infty}([0,1] ; W)} \leqslant\left\|g^{\prime}\right\|_{L^{\infty}([0,1] ; W)}+\|\Delta g\|_{L^{\infty}([0,1] ; W)}+\left\|\Delta^{2} g\right\|_{L^{\infty}([0,1] ; W)} .
$$

From the formula of $g$, since $f \in \mathcal{S}$ we can see easily that $F^{\prime \prime} \in L^{\infty}([0,1] ; W)$ (uniformly in $h \in(0,1])$. We obtain

$$
\|F(s)-s g(0)\|_{W} \leqslant C s^{2},
$$

where $C$ is a positive constant independent of $s$ (it depends on $f$ and $\gamma$ ). Thus,

$$
\|F(s)\|_{W}=s\|g(0)\|_{W}+\mathcal{O}\left(s^{2}\right) .
$$

In particular, for all $t, h>0$

$$
\left\|D(f)\left(t h^{2}\right)\right\|_{W}=\left\|F\left(t h^{2}\right)\right\|_{W}=t h^{2}\|g(0)\|_{W}+\mathcal{O}\left(t^{2} h^{4}\right) .
$$

This implies that

$$
\left\|D\left(f^{h}\right)(t)\right\|_{W}=\frac{1}{h^{d-\gamma+2}}\left\|D(f)\left(t h^{2}\right)\right\|_{W}=\frac{t}{h^{d-\gamma}}\|g(0)\|_{W}+\frac{1}{h^{d-\gamma}} \mathcal{O}\left(t^{2} h^{2}\right) .
$$

Fix $t>0$ :

$$
\lim _{h \rightarrow 0^{+}} \frac{t}{h^{d-\gamma}}\|g(0)\|_{W}+\frac{1}{h^{d-\gamma}} \mathcal{O}\left(t^{2} h^{2}\right)=+\infty
$$


We deduce that for $h>0$ sufficiently close to 0 ,

$$
\left\|D\left(f^{h}\right)(t)\right\|_{W}>C\|f\|_{W}^{3} .
$$

This contradicts (4.1), and Theorem 1.3 follows.

\section{Proof of Theorem 1.6}

We decompose the proof of Theorem 1.6 according to the three cases considered.

5.1. Well-posedness in $L^{2} \cap W$. We assume that $K$ is such that $\widehat{K} \in L^{p}$ for some $p \in[1, \infty]$, and we consider an initial data $u_{0} \in L^{2} \cap W$. For $T>0$ we define the following space:

$$
E_{T}=\left\{u \in C\left([0, T] ; L^{2} \cap W\right), \quad\|u\|_{L^{\infty}\left([0, T] ; L^{2} \cap W\right)} \leqslant 2\left\|u_{0}\right\|_{L^{2} \cap W}\right\} .
$$

It is a complete space metric when equipped with the metric

$$
d(u, v)=\|u-v\|_{L^{\infty}\left([0, T] ; L^{2} \cap W\right)} .
$$

Lemma 5.1. Let $p \in[1, \infty]$ and $K$ be such that $\widehat{K} \in L^{p}$. There exists $C$ such that for all $f, g \in L^{2} \cap W$,

$$
\|K *(f g)\|_{W} \leqslant C\|\widehat{K}\|_{L^{p}}\|f\|_{L^{2} \cap W}\|g\|_{L^{2} \cap W} .
$$

Proof. Let $f, g \in L^{2} \cap W$. We denote by $p^{\prime}$ the Hölder conjugate exponent of $p$. We have

$$
\begin{aligned}
\|K *(f g)\|_{W}=(2 \pi)^{d / 2}\|\widehat{K} \widehat{f g}\|_{L^{1}} & \leqslant C\|\widehat{K}\|_{L^{p}}\|\widehat{f g}\|_{L^{p^{\prime}}} \leqslant C\|\widehat{K}\|_{L^{p}}\|\widehat{f} * \widehat{g}\|_{L^{p^{\prime}}} \\
& \leqslant C\|\widehat{K}\|_{L^{p}}\|\widehat{f}\|_{L^{q}}\|\widehat{g}\|_{L^{q}},
\end{aligned}
$$

where $1+\frac{1}{p^{\prime}}=\frac{2}{q}$. Since $q \in[1,2]$ there exists $\theta \in[0,1]$ such that $\frac{1}{2}+\frac{\theta}{2}=\frac{1}{q}$ and

$$
\|\widehat{f}\|_{L^{q}} \leqslant\|\widehat{f}\|_{L^{1}}^{\theta}\|\widehat{f}\|_{L^{2}}^{1-\theta}=\|f\|_{W}^{\theta}\|f\|_{L^{2}}^{1-\theta} \leqslant\|f\|_{L^{2} \cap W} .
$$

The lemma follows.

Let $\Phi$ be as defined in Section 2, In view of the previous lemma, we have, for $u \in E_{T}$ and $t \in[0, T]$,

$$
\begin{aligned}
\|\Phi(u)(t)\|_{L^{2} \cap W} & \leqslant\left\|u_{0}\right\|_{L^{2} \cap W}+\int_{0}^{t}\left\|K *|u(\tau)|^{2} u(\tau)\right\|_{L^{2} \cap W} \mathrm{~d} \tau \\
& \leqslant\left\|u_{0}\right\|_{L^{2} \cap W}+\int_{0}^{t}\left\|K *|u(\tau)|^{2}\right\|_{W}\|u(\tau)\|_{L^{2} \cap W} \mathrm{~d} \tau \\
& \leqslant\left\|u_{0}\right\|_{L^{2} \cap W}+C\|\widehat{K}\|_{L^{p}} \int_{0}^{t}\|u(\tau)\|_{L^{2} \cap W}^{3} \mathrm{~d} \tau
\end{aligned}
$$

We obtain

$$
\|\Phi(u)(t)\|_{L^{\infty}\left([0, T] ; L^{2} \cap W\right)} \leqslant\left\|u_{0}\right\|_{L^{2} \cap W}+C\|\widehat{K}\|_{L^{p}}\left\|u_{0}\right\|_{L^{\infty}\left([0, T] ; L^{2} \cap W\right)}^{3} T .
$$


For $T$ sufficiently small (depending on $\left\|u_{0}\right\|_{L^{2} \cap W}$ ) $\|\Phi(u)\|_{L_{T}^{\infty} L^{2}} \leqslant 2\left\|u_{0}\right\|_{L^{2} \cap W}$. Let $u, v \in E_{T}$. From Lemma 5.1] we have

$$
\begin{aligned}
\| & \Phi(u)(t)-\Phi(v)(t) \|_{L^{2} \cap W} \\
\leqslant & \int_{0}^{t}\left\|K *\left(|u(\tau)|^{2}-|v(\tau)|^{2}\right) u(\tau)\right\|_{L^{2} \cap W} \mathrm{~d} \tau \\
& +\int_{0}^{t}\left\|K *|v(\tau)|^{2}(u(\tau)-v(\tau))\right\|_{L^{2} \cap W} \mathrm{~d} \tau \\
\leqslant & \int_{0}^{t}\left\|K *\left(|u(\tau)|^{2}-|v(\tau)|^{2}\right)\right\|_{W}\|u(\tau)\|_{L^{2} \cap W} \mathrm{~d} \tau \\
& +\int_{0}^{t}\left\|K *|v(\tau)|^{2}\right\|_{W}\|u(\tau)-v(\tau)\|_{L^{2} \cap W} \mathrm{~d} \tau \\
\leqslant & \int_{0}^{t} C\|\widehat{K}\|_{L^{p}}\left(\|u(\tau)\|_{L^{2} \cap W}^{2}+\|v(\tau)\|_{L^{2} \cap W}^{2}\right)\|u(\tau)-v(\tau)\|_{L^{2} \cap W} \mathrm{~d} \tau .
\end{aligned}
$$

We deduce that

$$
\|\Phi(u)-\Phi(v)\|_{L^{\infty}\left([0, T] ; L^{2} \cap W\right)} \leqslant C\|\widehat{K}\|_{L^{p}}\left\|u_{0}\right\|_{L^{2} \cap W}^{2} T\|u-v\|_{L^{\infty}\left([0, T] ; L^{2} \cap W\right)} .
$$

For $T$ possibly smaller (still depending on $\left\|u_{0}\right\|_{L^{2} \cap W}$ ) $\Phi$ is a contraction from $E_{T}$ to $E_{T}$, so it admits a unique fixed point in $E_{T}$, which is a solution for the Cauchy problem. By resuming the same arguments as in Proposition 3.2 we deduce that the fixed point obtained before is the unique solution for the Cauchy problem.

For $p=1$, the solution constructed before is global in time. In view of the conservation of the $L^{2}$-norm, we have

$$
\begin{aligned}
\|u(t)\|_{W} & \leqslant\left\|u_{0}\right\|_{W}+\int_{0}^{t}\left\|K *|u(\tau)|^{2}\right\|_{W}\|u(\tau)\|_{L^{2} \cap W} \mathrm{~d} \tau \\
& \leqslant\left\|u_{0}\right\|_{W}+\int_{0}^{t} C\|\widehat{K}\|_{L^{1}}\|u(\tau)\|_{L^{2}}^{2}\|u(\tau)\|_{W} \mathrm{~d} \tau \\
& \leqslant\left\|u_{0}\right\|_{W}+C\|\widehat{K}\|_{L^{1}}\left\|u_{0}\right\|_{L^{2}}^{2} \int_{0}^{t}\|u(\tau)\|_{W} \mathrm{~d} \tau
\end{aligned}
$$

and by the Gronwall lemma, we conclude that $\|u(t)\|_{W}$ remains bounded on finite time intervals. This completes the proof of the first point in Theorem 1.6.

5.2. Well-posedness in $W$. Let $\widehat{K} \in L^{\infty}$ and consider the Cauchy problem (1.1) with an initial data in $W$. For $T>0$ we define the following space:

$$
Y_{T}=\left\{u \in C([0, T] ; W),\|u\|_{L^{\infty}([0, T] ; W)} \leqslant 2\left\|u_{0}\right\|_{W}\right\} .
$$

The latter is a complete metric space when equipped with the metric

$$
d(u, v)=\|u-v\|_{L^{\infty}([0, T] ; W)} .
$$

As previously, the local existence of a solution is easily shown by a fixed point argument, since $\Phi$ is a contraction from $Y_{T}$ to $Y_{T}$, and we show that it is unique. The proof relies on the following lemma:

Lemma 5.2. There exists $C$ such that for all $f, g \in W$,

$$
\|K *(f g)\|_{W} \leqslant C\|\widehat{K}\|_{L^{\infty}}\|f\|_{W}\|g\|_{W} .
$$


Proof. Let $f, g \in W$. We have

$$
\begin{aligned}
\|K *(f g)\|_{W}=(2 \pi)^{d / 2}\|\widehat{K} \widehat{f g}\|_{L^{1}} & \leqslant(2 \pi)^{d / 2}\|\widehat{K}\|_{L^{\infty}}\|\widehat{f} * \widehat{g}\|_{L^{1}} \\
& \leqslant(2 \pi)^{d / 2}\|\widehat{K}\|_{L^{\infty}}\|\widehat{f}\|_{L^{1}}\|\widehat{g}\|_{L^{1}} \\
& \leqslant(2 \pi)^{d / 2}\|\widehat{K}\|_{L^{\infty}}\|f\|_{W}\|g\|_{W} .
\end{aligned}
$$

It then suffices to reproduce the proof given in the previous subsection in order to prove the second point of Theorem 1.6 by replacing Lemma 5.1 with Lemma 5.2 .

5.3. Ill-posedness in $W$. For $\gamma \in(0, d)$ we consider the Cauchy problem (1.1) with the kernel $K$ given by its Fourier transform

$$
\widehat{K}(\xi)=\frac{1}{|\xi|^{d-\gamma}} \mathbf{1}_{\{|\xi| \leqslant 1\}} .
$$

Then, $\widehat{K} \in L^{p}$ for all $p \in\left[1, \frac{d}{d-\gamma}\right)$. Conversely, for $p \in[1, \infty)$, we can always find $\gamma \in(0, d)$ such that $K$, defined by (5.1), satisfies $\widehat{K} \in L^{p}$.

From the first point of Theorem 1.6, the Cauchy problem (1.1) is locally wellposed in $L^{2} \cap W$. We suppose that (1.1) is well-posed in $W$ : let $T>0$ be the local time existence of the solution. Arguing as in Section 4 , this implies that there exists $C>0$ such that for all $f \in W$ and all $t \in[0, T]$,

$$
\|D(f)(t)\|_{W} \leqslant C\|f\|_{W}^{3},
$$

where $D$ is the operator defined in Section 4 . Let $f \in \mathcal{S}\left(\mathbf{R}^{d}\right)$. As in Section 4 we define the family of functions $\left(f^{h}\right)_{0<h \leqslant 1}$ by

$$
f^{h}(x)=f(h x) .
$$

From (5.2) we also have for all $t \in[0, T]$,

$$
\left\|D\left(f^{h}\right)(t)\right\|_{W} \leqslant C\left\|f^{h}\right\|_{W}^{3}=C\|f\|_{W}^{3} .
$$

We define $K_{h}$ by setting $\widehat{K_{h}}(\xi)=\frac{1}{|\xi|^{d-\gamma}} \mathbf{1}_{\left\{|\xi|>\frac{1}{h}\right\}}$. We use the following identity:

$$
\begin{aligned}
& \mathcal{F}\left(K *\left|U(\tau) f^{h}\right|^{2} U(\tau) f^{h}\right)(\xi) \\
& =\frac{(2 \pi)^{d / 2}}{h^{3 d}} \iint_{\mathbf{R}^{2 d}} e^{i \tau|\xi-y|^{2}} e^{i \tau|y-z|^{2}} e^{-i \tau|z|^{2}} \widehat{K}(y) \widehat{f}\left(\frac{\xi-y}{h}\right) \widehat{\bar{f}}\left(\frac{z}{h}\right) \widehat{f}\left(\frac{y-z}{h}\right) \mathrm{d} y \mathrm{~d} z \\
& =\frac{(2 \pi)^{d / 2}}{h^{3 d}} \iint_{\mathbf{R}^{2 d}} e^{i \tau|\xi-y|^{2}} e^{i \tau|y-z|^{2}} e^{-i \tau|z|^{2}} \frac{1}{|y|^{d-\gamma}} \widehat{f}\left(\frac{\xi-y}{h}\right) \widehat{\bar{f}}\left(\frac{z}{h}\right) \widehat{f}\left(\frac{y-z}{h}\right) \mathrm{d} y \mathrm{~d} z \\
& -\frac{(2 \pi)^{d / 2}}{h^{3 d}} \iint_{\mathbf{R}^{2 d}} e^{i \tau|\xi-y|^{2}} e^{i \tau|y-z|^{2}} e^{-i \tau|z|^{2}} \widehat{K}_{|y|>1}(y) \widehat{f}\left(\frac{\xi-y}{h}\right) \widehat{\bar{f}}\left(\frac{z}{h}\right) \widehat{f}\left(\frac{y-z}{h}\right) \mathrm{d} y \mathrm{~d} z .
\end{aligned}
$$

We inject the above formula into the expression of $\left\|D\left(f^{h}\right)(t)\right\|_{W}$ and perform the same changes of variables as in Section 4 . We obtain

where

$$
\left\|D\left(f^{h}\right)(t)\right\|_{W} \geqslant \frac{t}{h^{d-\gamma}}\left(\left\|\left(K *|f|^{2}\right) f\right\|_{W}+\mathcal{O}\left(t h^{2}\right)\right)-\frac{1}{h^{d-\gamma+2}} X^{h},
$$

$$
X^{h}=\left\|\int_{0}^{t h^{2}} U(t-\tau)\left(K_{h} *|U(\tau) f|^{2} U(\tau) f\right) \mathrm{d} \tau\right\|_{W}
$$


For $q \in\left(\frac{d}{d-\gamma}, \infty\right)$,

$$
\begin{aligned}
X^{h} & \leqslant \int_{0}^{t h^{2}}\left\|\left(K_{h} *|U(\tau) f|^{2}\right) U(\tau) f\right\|_{W} \mathrm{~d} \tau \leqslant \int_{0}^{t h^{2}}\left\|K_{h} *|U(\tau) f|^{2}\right\|_{W}\|U(\tau) f\|_{W} \mathrm{~d} \tau \\
& \leqslant\|f\|_{W} \int_{0}^{t h^{2}}\left\|\widehat{K_{h}}\right\|_{L^{q}}\left\|\mathcal{F}\left(|U(\tau) f|^{2}\right)\right\|_{L^{q^{\prime}}} \mathrm{d} \tau \leqslant C t h^{2}\left\|\widehat{K_{h}}\right\|_{L^{q}},
\end{aligned}
$$

for some constant $C$ independent of $h \in(0,1]$ and $t \in[0, T]$ (recall that $f \in \mathcal{S}\left(\mathbf{R}^{d}\right)$ ). Moreover,

$$
\left\|\widehat{K_{h}}\right\|_{L^{q}}=\left(\int_{\mathbf{R}^{d}} \frac{1}{|y|^{(d-\gamma) q}} \mathbf{1}_{\left\{|y|>\frac{1}{h}\right\}} \mathrm{d} y\right)^{1 / q}=C\left(\int_{1 / h}^{+\infty} \frac{r^{d-1}}{r^{(d-\gamma) q}} \mathrm{~d} r\right)^{1 / q},
$$

so $\left\|\widehat{K_{h}}\right\|_{L^{q}}=C h^{d-\gamma-\frac{d}{q}}$ and

$$
\left\|D\left(f^{h}\right)(t)\right\|_{W} \geqslant \frac{t}{h^{d-\gamma}}\left(\left\|\left(K *|f|^{2}\right) f\right\|_{W}+\mathcal{O}\left(t h^{2}\right)-C h^{d-\gamma-\frac{d}{q}}\right) .
$$

Fix $t>0$ :

$$
\lim _{h \rightarrow+\infty} \frac{t}{h^{d-\gamma}}\left(\left\|\left(K *|f|^{2}\right) f\right\|_{W}+\mathcal{O}\left(t h^{2}\right)-C h^{d-\gamma-\frac{d}{q}}\right)=+\infty .
$$

So, for $h>0$ small enough we have

$$
\left\|D\left(f^{h}\right)(t)\right\|_{W}>C\|f\|_{W}^{3},
$$

hence a contradiction.

\section{REFERENCES}

[1] Hajer Bahouri, Jean-Yves Chemin, and Raphaël Danchin, Fourier analysis and nonlinear partial differential equations, Grundlehren der Mathematischen Wissenschaften [Fundamental Principles of Mathematical Sciences], vol. 343, Springer, Heidelberg, 2011. MR2768550 (2011m:35004)

[2] Ioan Bejenaru and Terence Tao, Sharp well-posedness and ill-posedness results for a quadratic non-linear Schrödinger equation, J. Funct. Anal. 233 (2006), no. 1, 228-259, DOI 10.1016/j.jfa.2005.08.004. MR2204680(2007i:35216)

[3] N. Burq, P. Gérard, and N. Tzvetkov, An instability property of the nonlinear Schrödinger equation on $S^{d}$, Math. Res. Lett. 9 (2002), no. 2-3, 323-335. MR1909648 (2003c:35144)

[4] Rémi Carles, Eric Dumas, and Christof Sparber, Multiphase weakly nonlinear geometric optics for Schrödinger equations, SIAM J. Math. Anal. 42 (2010), no. 1, 489-518, DOI 10.1137/090750871. MR2607351(2011c:35529)

[5] Rémi Carles, Eric Dumas, and Christof Sparber, Geometric optics and instability for NLS and Davey-Stewartson models, J. Eur. Math. Soc. (JEMS) 14 (2012), no. 6, 1885-1921, DOI 10.4171/JEMS/350. MR2984591

[6] Thierry Cazenave, Semilinear Schrödinger equations, Courant Lecture Notes in Mathematics, vol. 10, New York University, Courant Institute of Mathematical Sciences, New York, 2003. MR2002047(2004j:35266)

[7] M. Christ, Power series solution of a nonlinear Schrödinger equation, Mathematical aspects of nonlinear dispersive equations, Ann. of Math. Stud., vol. 163, Princeton Univ. Press, Princeton, NJ, 2007, pp. 131-155. MR2333210(2008k:35438)

[8] M. Christ, J. Colliander, and T. Tao, Instability of the periodic nonlinear Schrödinger equation, archived as http://arxiv.org/abs/math.AP/0311227.

[9] Mathieu Colin and David Lannes, Short pulses approximations in dispersive media, SIAM J. Math. Anal. 41 (2009), no. 2, 708-732, DOI 10.1137/070711724. MR2515782 (2010e:35179)

[10] James Colliander and Tadahiro Oh, Almost sure well-posedness of the cubic nonlinear Schrödinger equation below $L^{2}(\mathbb{T})$, Duke Math. J. 161 (2012), no. 3, 367-414, DOI 10.1215/00127094-1507400. MR2881226 
[11] Elena Cordero and Fabio Nicola, Strichartz estimates in Wiener amalgam spaces for the Schrödinger equation, Math. Nachr. 281 (2008), no. 1, 25-41, DOI 10.1002/mana.200610585. MR2376466 (2008k:35387)

[12] Elena Cordero and Davide Zucco, Strichartz estimates for the Schrödinger equation (English, with English and Spanish summaries), Cubo 12 (2010), no. 3, 213-239. MR2779383 (2012c:35368)

[13] Johannes Giannoulis, Alexander Mielke, and Christof Sparber, High-frequency averaging in semi-classical Hartree-type equations, Asymptot. Anal. 70 (2010), no. 1-2, 87-100. MR2731651(2012c:35412)

[14] J. Ginibre and G. Velo, The global Cauchy problem for the nonlinear Schrödinger equation revisited (English, with French summary), Ann. Inst. H. Poincaré Anal. Non Linéaire 2 (1985), no. 4, 309-327. MR801582 (87b:35150)

[15] Ryosuke Hyakuna and Masayoshi Tsutsumi, On existence of global solutions of Schrödinger equations with subcritical nonlinearity for $\widehat{L}^{p}$-initial data, Proc. Amer. Math. Soc. 140 (2012), no. 11, 3905-3920, DOI 10.1090/S0002-9939-2012-11314-0. MR2944731

[16] J.-L. Joly, G. Métivier, and J. Rauch, Coherent nonlinear waves and the Wiener algebra (English, with English and French summaries), Ann. Inst. Fourier (Grenoble) 44 (1994), no. 1, 167-196. MR1262884 (95c:35163)

[17] Tosio Kato, On nonlinear Schrödinger equations (English, with French summary), Ann. Inst. H. Poincaré Phys. Théor. 46 (1987), no. 1, 113-129. MR877998 (88f:35133)

[18] Markus Keel and Terence Tao, Endpoint Strichartz estimates, Amer. J. Math. 120 (1998), no. 5, 955-980. MR1646048 (2000d:35018)

[19] David Lannes, Nonlinear geometrical optics for oscillatory wave trains with a continuous oscillatory spectrum, Adv. Differential Equations 6 (2001), no. 6, 731-768. MR.1829094 (2002a:35190)

[20] Luc Molinet, On ill-posedness for the one-dimensional periodic cubic Schrodinger equation, Math. Res. Lett. 16 (2009), no. 1, 111-120. MR2480565 (2010b:35440)

[21] L. Mouzaoui, High-frequency averaging in semi-classical singular Hartree equations, Asymptot. Anal. 84 (2013), no. 3-4, 229-245. MR 3136109

[22] Tadahiro Oh and Catherine Sulem, On the one-dimensional cubic nonlinear Schrödinger equation below $L^{2}$, Kyoto J. Math. 52 (2012), no. 1, 99-115, DOI 10.1215/21562261-1503772. MR2892769

[23] Jeffrey Rauch, Hyperbolic partial differential equations and geometric optics, Graduate Studies in Mathematics, vol. 133, American Mathematical Society, Providence, RI, 2012. MR.2918544

Mathématiques, CC 051, CNRS and Université Montpellier 2, 34095 Montpellier, FRANCE

E-mail address: Remi.Carles@math.cnrs.fr

Mathématiques, CC 051, CNRS And Université Montpellier 2, 34095 Montpellier, FRANCE

E-mail address: lounes.mouzaoui@univ-montp2.fr 